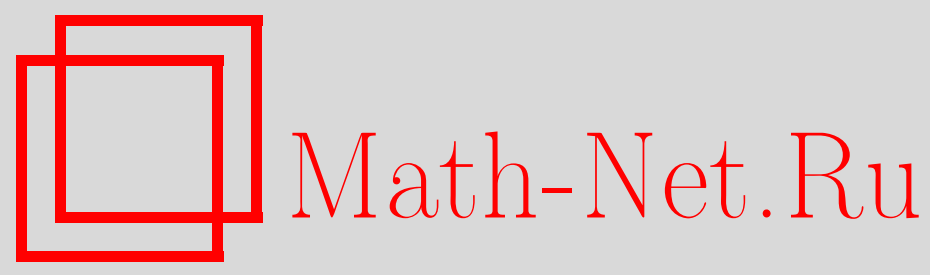

П. И. Топалов, Тензорные инварианты натуральных механических систем на компактных поверхностях и соответствующие им интегралы, Матем. заметки, 1999, том 66, выпуск 3, 417-430

DOI: https://doi.org/10.4213/mzm1183

Использование Общероссийского математического портала Math-Net.Ru подразумевает, что вы прочитали и согласны с пользовательским соглашением http://www . mathnet.ru/rus/agreement

Параметры загрузки:

IP: 3.85 .73 .92

26 апреля 2023 г., 13:42:07

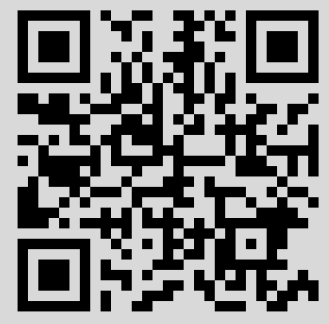




\section{ТЕНЗОРНЫЕ ИНВАРИАНТЫ НАТУРАЛЬНЫХ МЕХАНИЧЕСКИХ СИСТЕМ НА КОМПАКТНЫХ ПОВЕРХНОСТЯХ И СООТВЕТСТВУЮЩИЕ ИМ ИНТЕГРАЛЫ}

\section{П. И. Топалов}

В работе рассматривается задача классификации тензорных инвариантов геодезических потоков на компактных поверхностях. K таким инвариантам относятся первые интегралы, многозначные интегралы и поля симметрии. Указанная задача решается для тензорньх инвариантов произвольной степени. Показано, что если такой инвариант где-то обращается в нуль, то имеется двузначное поле симметрии и многозначный интеграл.

Библиограффия: 5 названий.

1. Введение. Тензорным инвариантом динамической системы $X$ на многообразии $M$ называется ненулевое гладкое тензорное поле $T$ такое, что $L_{X} T=0$, где $L_{X}-$ производная Ли. В частности, любой интеграл (т.е. скалярная гладкая функция $f$, инвариантная относительно потока $X$ ) является тензорньм инвариантом. Для любой динамической системы $X$ на любом многообразии $M$ всегда имеются тензорные инварианты, например - это само поле $X$, единичньй оператор $1 \in \operatorname{Hom}(\mathcal{T} M, \mathcal{T} M)$ и их тензорные произведения. С другой стороны, заметим, что если $f$ нетривиальньй интеграл (т.е. $f \neq$ const), то все тензорные произведения $d f \otimes \cdots \otimes d f$ являются тензорными инвариантами. Следовательно, если система $X$ имеет интеграл, то существуют тензорные инварианты любого типа $(m, n)$.

Возникает обратный вопрос. Когда из существования тензорного инварианта (отличного от интеграла) следует существование нетривиальных интегралов или, хотя бы, многозначных интегралов динамической системы? В такой формулировке вопрос впервые был поставлен и изучался В. В. Козловым и С. В. Болотиным в [1], [2], где было доказано, что в случае геодезического потока на компактной поверхности существование нетривиального поля симметрий влечет существование ненулевого многозначного интеграла. Поле симметрии назьвается “нетривиальны.м", если оно не равно const $D_{1}$, где $D_{1}$ - геодезическое векторноеполе. Многозначным интегралом динамической системы $X$ называется ненулевая замкнутая 1 -форма $\alpha$ такая, что $\alpha(X)=0$.

Существует общий подход, которьй всегда позволяет найти по тензорному инварианту $T$ некоторые интегралы, но они могут оказаться тривиальными. Действительно, пусть $V$ - векторное пространство. Рассмотрим естественное действие $\rho: G L(V) \rightarrow$

Работа выполнена при финансовой поддержке Российского фонда фундаментальных исследований, и Международного научного фонда. 
$G L\left(V \otimes \cdots \otimes V^{*}\right)$, которое в некотором изначально фиксированном базисе $\left\{e_{i}\right\}$ на $V$ задается формулой,

$$
[\rho(a)(T)]_{l_{1} \ldots l_{n}}^{k_{1} \ldots k_{m}} \stackrel{\text { def }}{=} a_{i_{1}}^{k_{1}} \ldots a_{i_{m}}^{k_{m}} T_{j_{1} \ldots j_{n}}^{i_{1} \ldots i_{m}} \tilde{a}_{l_{1}}^{j_{1}} \ldots \tilde{a}_{l_{n}}^{j_{n}}
$$

где $a_{j}^{i} \tilde{a}_{k}^{j}=\delta_{k}^{i}$. Рассмотрим инварианты этого действия $\left\{I_{\alpha}\right\}_{\alpha}$ как функции от координат $\left(T_{j_{1} \ldots j_{n}}^{i_{1} \ldots i_{m}}\right)$. Тогда если $T$ - тензорньй инвариант динамической системы $X$, то любому инварианту $I_{\alpha}$ действия $\rho$ соответствует интеграл $I_{\alpha}(x) \stackrel{\text { def }}{=} I_{\alpha}(T(x))$.

2. Обозначения и вводные результаты. Рассмотрим двумерное замкнутое компактное и ориентированное многообразие $M$. Пусть на нем задана риманова метрика $g$. Геодезический поток этой метрики является автономной динамической системой на касательном расслоении $\mathcal{T} M \stackrel{\pi}{\rightarrow} M$. Метрика $g$, рассматриваемая как функция на $\mathcal{T} M$, является интегралом геодезического потока. Рассмотрим изоэнергетическую поверхность этого интеграла $Q^{3} \stackrel{\text { def }}{=}\{g(v, v)=1\}$. На $Q^{3}$ определим векторные поля $D_{0}, D_{1}$ и $D_{2}$, линейно независимые в любой точке.

На $Q^{3}$ существует естественное действие окружности $S^{1}=\{\varphi \bmod 2 \pi\}$, которое задается формулой

$$
\rho_{\varphi}: v \mapsto\{\text { поворот } v \text { на угол }+\varphi\} .
$$

Фундаментальное векторное поле этого действия обозначим через $D_{0}$, т.е.

$$
\left.D_{0}(v) \stackrel{\text { def }}{=} \frac{d}{d \varphi}\right|_{\varphi=0}\left[\rho_{\varphi}(v)\right] .
$$

Обозначим через $D_{1}$ геодезическое векторное поле. Тогда векторное поле $D_{2}$ определим формулой $D_{2} \stackrel{\text { def }}{=}\left[D_{0}, D_{1}\right]$.

Имеют место коммутационные соотношения

$$
\left\{\begin{array}{l}
{\left[D_{0}, D_{1}\right]=D_{2},} \\
{\left[D_{0}, D_{2}\right]=-D_{1},} \\
{\left[D_{1}, D_{2}\right]=K D_{0},}
\end{array}\right.
$$

где $K$ обозначает гауссову кривизну, которую мы подняли на $Q^{3}$.

Обозначим через $\left(T, \Theta^{1}, \Theta^{2}\right)$ корепер, двойственньй к $\left(D_{0}, D_{1}, D_{2}\right)$, т.е. $T\left(D_{0}\right)=1$, $T\left(D_{1}\right)=0$ и т.д. В классических терминах на $\mathcal{T}^{*} M$ после применения преобразования Лежандра форма $\Theta^{1}$ переходит в форму $\left.(p d q)\right|_{Q^{3}}$. Через $\omega_{g}$ будем обозначать формy $d \Theta^{1}$.

Имеют место соотношения

$$
\left\{\begin{aligned}
d T & =-K \Theta^{1} \wedge \Theta^{2} \\
d \Theta^{1} & =T \wedge \Theta^{2} \\
d \Theta^{2} & =\Theta^{1} \wedge T
\end{aligned}\right.
$$

Соотношения $(*)$ и $(* *)$ являются следствием структурных уравнений метрической связности. В частности, из $(* *)$ получаем, что $L_{D_{1}} T=-K \Theta^{2}, L_{D_{1}} \Theta^{1}=0, L_{D_{1}} \Theta^{2}=T$.

Докажем сначала несколько утверждений. 
УТВЕРЖДЕНИЕ 1. Гладкое векторное поле $\vec{X}$ является полем симметрий геодезического потока тогда и только тогда, когда существует гладкая функция $x \in C^{\infty}\left(Q^{3}\right)$ такая, что

$$
\left\{\begin{array}{l}
\vec{X}=x D_{2}+\dot{x} D_{0}+I D_{1}, \\
\ddot{x}+K x=0,
\end{array}\right.
$$

где $\dot{x}$ обозначает производную вдоль векторного поля $D_{1}$, а I есть некоторый интеграл геодезического потока.

ДокАЗАТЕЛЬСтво. Пусть $X$ есть гладкое векторное поле на $Q^{3}$. Тогда существуют гладкие функции $x, y$ и $z \in C^{\infty}\left(Q^{3}\right)$ такие, что

$$
X=x D_{2}+y D_{0}+z D_{1} .
$$

С другой стороны, из $(*)$ получаем, что

$$
\begin{aligned}
{\left[D_{1}, X\right] } & =\dot{x} D_{2}+\dot{y} D_{0}+\dot{z} D_{1}+x K D_{0}-y D_{2} \\
& =(\dot{x}-y) D_{2}+(\dot{y}+K x) D_{0}+\dot{z} D_{1} .
\end{aligned}
$$

Следовательно, $\left[D_{1}, X\right]=0$ тогда и только тог да, когда функции $x, y$ и $z$ удовлетворяют системе

$$
\left\{\begin{array}{l}
\dot{z}=0, \\
y=\dot{x}, \\
\ddot{x}+K x=0 .
\end{array}\right.
$$

Тем самьп, утверждение доказано.

Интеграл $I$, которьй стоит в условии этого утверждения, в некотором смысле является “нётеровьм" (см. [3]). Действительно, согласно теореме Нётера любая однопараметрическая группа изометрий на $M$ задает гладкое нетривиальное поле симметрии $X$ на $Q^{3}$, которое сохраняет форму $\Theta^{1}$. Следовательно, имеем $0=L_{X} \Theta^{1}=i_{X} \omega+d I$. Таким образом, получаем, что $d I \neq 0$ везде, где $X$ не пропорционально геодезическому векторному полю. Следовательно, в этом случае $I$ - нетривиальньй интеграл, совпадающий с классическим интегралом Нётера.

Совершенно аналогично доказьваются

УТВЕРЖДЕНИЕ 2. Гладкая 1-форма $\alpha$ является тензорным инвариантом геодезического потока тогда и только тогда, когда существует гладкая функиия $x \in C^{\infty}\left(Q^{3}\right)$ такая, что

$$
\left\{\begin{array}{l}
\alpha=x T-\dot{x} \Theta^{2}+I \Theta^{1}, \\
\ddot{x}+K x=0,
\end{array}\right.
$$

где I - некоторый интеграл геодезического потока.

СлЕДСТВИЕ 1. Если род поверхности $\geqslant 1 u K \leqslant 0$, то поле симметрий геодезического потока в области, где $K<0$, облзательно будет пропориионально геодезическому векторному полю $D_{1}$. 
УТВЕРЖДЕНИЕ 3. Форма $\alpha$ является многозначным интегралом геодезического потока тогда и только тогда, когда существует гладкая функиия $x$ на $Q^{3}$ такая, что

$$
\left\{\begin{array}{l}
\alpha=x T-\dot{x} \Theta^{2} \\
\ddot{x}+K x=0 \\
(\dot{x})_{0}+x_{2}=0
\end{array}\right.
$$

əдe $(\dot{x})_{0} \stackrel{\text { def }}{=} D_{0} D_{1} x u x_{2} \stackrel{\text { def }}{=} D_{2} x$.

ТЕОрема 1. Пусть $\omega^{2}$ - есть тензорный инвариант типа $(0,2)$, который не является линейной комбинацией с постоянными коэффициентами форм $\Theta^{1} \otimes \Theta^{1}$ и $T \wedge \Theta^{2}$. Тогда если $\omega^{2}\left(D_{0}, D_{0}\right)=0$, то существует нетривиальное поле симметрии геодезического потока.

ДокАЗАТЕЛЬСТво. Любой тензор типа $(0,2)$ можно записать в виде

$$
\begin{aligned}
\omega^{2}= & f \Theta^{1} \otimes \Theta^{1}+\left(\Theta^{1} \otimes \alpha^{1}+\beta^{1} \otimes \Theta^{1}\right) \\
& +b_{00} T \otimes T+b_{01} T \otimes \Theta^{2}+b_{10} \Theta^{2} \otimes T+b_{11} \Theta^{2} \otimes \Theta^{2} .
\end{aligned}
$$

Так как $\Theta^{1}$ инвариантна относительно геодезического потока, то $\alpha^{1}$ и $\beta^{1}$ являются тензорньми инвариантами, а $f$ является интегралом. С другой стороны, из первого уравнения системы $(* * *)$ находим, что $b_{10}+b_{01}=0$. Тогда, складьвая второе и третье уравнение той же системы, получаем, что $b_{11}=0$. Из второго уравнения видно, что $\dot{b}_{01}=0$, т.е. $b_{01}-$ интеграл.

Допустим, что все полученные поля симметрий нулевые, а интегралы - константы. Тогда $\omega^{2}=c_{1} \Theta^{1} \otimes \Theta^{1}+c_{2} T \wedge \Theta^{2}$, где $c_{1}$ и $c_{2}$ константы. Но это противоречит условию теоремы. Следовательно, имеется нетривиальное поле симметрии. Теорема доказана.

Из этой теоремы, в частности, следует, что если тензорный инвариант есть 2-форма, не являющаяся линейной комбинацией с постоянными коэффициентами форм $\Theta^{1} \otimes \Theta^{1}$ и $\omega_{g}$, то существует нетривиальное поле симметрии геодезического потока.

Таким же образом доказывается

ТЕОрема 2. Пусть $\omega^{3}$ есть тензорный инвариант типа $(0,3)$, который порохдается формами $T$ и $\Theta^{2}$ и такой, что $\omega^{3}\left(D_{0}, D_{0}, D_{0}\right)=0$. Тогда существует нетривиальное поле симметрий геодезического потока.

3. Приложения к группе траекторных автоморфизмов геодезического потока. Пусть $M^{2}$ - компактное связное замкнутое ориентируемое двумерное риманово многообразие. Рассмотрим группу гладких ориентированных диффеоморфизмов изоэнергетической поверхности $Q^{3}$, которые переводят траектории геодезического потока в траектории с сохранением их естественной ориентации.

ОпРЕДЕЛЕНИЕ 1. Определенную таким образом группу будем назьвать әруппой траекторных автоморфизмов геодезического потока.

ОпРЕДЕлЕниЕ 2. Группу гладких ориентированных диффеоморфизмов изоэнергетической поверхности $Q^{3}$, которые переводят геодезическое векторное поле в себя, будем называть группой автоморфизмов геодезического потока. 
Теорема 3. Любому несимплектическому траекторному автоморфизму геодезического потока соответствует нетривиальный интеграл.

СледСтвиЕ 2. Группа траекторных автоморфизмов неинтегрируемого геодезического потока состоит из симплектоморфизмов.

ДоКАЗАТЕЛЬСТВо ТЕОРЕМЫ 3. Без ограничения общности будем считать, что заданы два геодезических потока на двух разных поверхностях $M^{2}$ и $M^{2^{\prime}}$. Допустим, что диффеоморфизм $\phi$ является траекторньм диффеоморфизмом. Тогда,

$$
d_{x_{0}} \phi\left(D_{1}\left(x_{0}\right)\right)=a\left(x_{0}\right) D_{1}^{\prime}\left(\phi\left(x_{0}\right)\right),
$$

где $a$ - гладкая функция, $a>0$. Следовательно, имеют место соотношения,

$$
i_{D_{1}}\left(\phi^{*} \omega_{g^{\prime}}\right)_{x}=\omega_{g^{\prime}}(\phi(x))\left(d_{x} \phi\left(D_{1}(x)\right), d_{x} \phi(\cdot)\right)=0,
$$

так как $D_{1}^{\prime}$ лежит в ядре формы $\omega_{g^{\prime}}$. Отсюда следует, что $\left(\phi^{*} \omega_{g^{\prime}}\right)_{x}=I(x) \omega_{g}$, где $I(x)-$ гладкая функция. Следовательно,

$$
L_{D_{1}}\left(\phi^{*} \omega_{g^{\prime}}\right)=i_{D_{1}} d\left[\phi^{*} \omega_{g^{\prime}}\right]+d\left[i_{D_{1}} \phi^{*} \omega_{g^{\prime}}\right]=0 .
$$

В силу инвариантности формы $\omega_{g}$ получаем, что $I(x)$ является интегралом поля $D_{1}$. Заметим, что до сих пор мы не пользовались тем, что $D_{1}$ - геодезический поток.

С другой стороны, имеем, что

$$
i_{D_{1}}\left(\phi^{*} \Theta^{1^{\prime}}\right)_{x}=\Theta^{1^{\prime}}(\phi(x))\left(d_{x} \phi\left(D_{1}\right)\right)=a(x)
$$

и, следовательно,

$$
\left(\phi^{*} \Theta^{1^{\prime}}\right)_{x}=a(x) \Theta^{1}+p(x) T+q(x) \Theta^{2} .
$$

Отсюда получаем, что

$$
\begin{aligned}
d\left[\phi^{*} \Theta^{1^{\prime}}\right]= & d\left[a \Theta^{1}+p T+q \Theta^{2}\right] \\
= & d a \wedge \Theta^{1}+d p \wedge T+d q \wedge \Theta^{2} \\
& +a T \wedge \Theta^{2}-K p \Theta^{1} \wedge \Theta^{2}+q \Theta^{1} \wedge T \\
= & \left\{a-p_{2}+q_{0}\right\} \omega_{g}+\left\{a_{0}-p_{1}-q\right\} T \wedge \Theta^{1} \\
& +\left\{-a_{2}+q_{1}-K p\right\} \Theta^{1} \wedge \Theta^{2} .
\end{aligned}
$$

Из равенства $d\left[\phi^{*} \Theta^{1^{\prime}}\right]=I(x) \omega_{g}$ следует, что вьполняется система дифференциальных соотношений

$$
\left\{\begin{array}{l}
a(x)-p_{2}+q_{0}=I(x), \\
a_{0}-p_{1}-q=0, \\
-a_{2}+q_{1}-K p=0 .
\end{array}\right.
$$

Интегрируя первое равенство по элементу объема $\sigma_{g}$, находим

$$
\int_{Q^{3}} I \sigma_{g}=\int_{Q^{3}}\left\{a-p_{2}+q_{0}\right\} \sigma_{g}=\int_{Q^{3}} a \sigma_{g} .
$$


С другой стороны, имеем

$$
\phi^{*} \sigma_{g^{\prime}}=-\phi^{*}\left(\Theta^{1^{\prime}}\right) \wedge \phi^{*}\left(\omega_{g^{\prime}}\right)=a(x) I(x) \sigma_{g} .
$$

Теперь докажем нашу теорему. Сначала предположим, что изначально задана одна поверхность, т.е. $M^{2}=M^{2^{\prime}}$ и $g=g^{\prime}$. Согласно уже доказанному имеет место равенство $\phi^{*} \omega_{g}=I \omega_{g}$, где $I(x)$ - интеграл геодезического потока. Допустим, что этот интеграл тривиален, т.е. $I(x)=c$, где $c$ есть некоторая константа. Тогда из равенств (1) и $(2)$ соответственно получаем

$$
\left\{\begin{aligned}
c \operatorname{Vol}\left(Q^{3}\right) & =\int_{Q^{3}} a \sigma_{g} \\
\operatorname{Vol}\left(Q^{3}\right) & =c \int_{Q^{3}} a \sigma_{g} .
\end{aligned}\right.
$$

Отсюда следует, что

$$
\int_{Q^{3}} a \sigma_{g}=c^{2} \int_{Q^{3}} a \sigma_{g} .
$$

С другой стороны, из второго равенства системы видно, что $\int_{Q^{3}} a \sigma_{g} \neq 0$. Следовательно, $c= \pm 1$. Но по определению $a(x)>0$, и из первого равенства системы получаем, что $c=1$. Это противоречит условию теоремы, что диффеоморфизм $\phi$ несимплектический. Тем самьм, теорема доказана.

Из доказательства этой теоремы можно усмотреть следующее утверждение

СлЕДСТвИЕ 3. Пусть $(M, g) u\left(M^{\prime}, g^{\prime}\right)$ - двумерные компактные и замкнутые римановы многообразия. Рассмотрим геодезические потоки на $Q^{3}$ и $Q^{3^{\prime}}$. Тогда если существует изоморфизм этих потоков, то обязательно $\operatorname{Vol}(M, g)=$ $\operatorname{Vol}\left(M^{\prime}, g^{\prime}\right)$.

ТЕОРемА 4. Пусть задан несимплектический траекторный автоморфизм натуральной механической системы с гамильтонианом

$$
H=\frac{1}{2} g^{i j}(\bar{x}) p_{i} p_{j}+U(\bar{x})
$$

(әде $\left\{\left(x^{i}, p_{i}\right)\right\}$ - “естественные” координаты на $\left.\mathcal{T}^{*} M\right)$ на изоәнергетической поверхности $Q_{h}^{3} \stackrel{\text { def }}{=}\left\{p \in \mathcal{T}^{*} M \mid H(p)=h\right\}$ в предположении, что $U<h$. Тогда существует нетривиальный интеграл системы на $Q_{h}^{3}$.

ДокАЗАТЕЛЬСтво. Теорема является следствием теоремы 3 и принципа Мопертюи.

Теорема 5. Геодезический поток на компактной поверхности имеет многозначный интеграл тогда и только тогда, когда существует автоморфизм, не сохраняющий форму $\Theta^{1}$ (в классических терминах это форма $\left.\left.(p d q)\right|_{Q^{3}}\right)$.

Пусть теперь задано нетривиальное поле симметрии $X$ геодезического потока. Без ограничения общности считаем, что $\Theta^{1}(X)=0$. Рассмотрим поток этого поля $\left\{g^{t}(x)\right\}$. Очевидно, что тогда диффеоморфизмы $g^{t}: Q^{3} \rightarrow Q^{3}$ являются автоморфизмами геодезического потока $D_{1}$. Следовательно, в этом случае получаем однопараметрическое семейство интегралов $\{I(t ; x)\}$, где $\left[g_{t}^{*} \omega_{g}\right](x)=I(t ; x) \omega_{g}(x)$, и однопараметрическое семейство инвариантных 1-форм $\left\{g_{t}^{*} \Theta^{1}\right\}$. Обозначим через $I(x)$ интеграл, заданньй равенством $d\left[\alpha_{X}\right]=I \omega_{g}$, где, как и раньше, $\alpha_{X} \stackrel{\text { def }}{=} i_{X} \omega_{g}$. 
Теорема 6. Имеют место соотношения:

a) $I(x)=\partial_{t} I(0 ; x)$,

б) $I(t, x)=\exp \{I(x) t\}$

в) $g_{t}^{*} \Theta^{1}=\Theta^{1}+\left[\int_{0}^{t} I(u ; x) d u\right] \alpha_{X}$.

Теорема 6 показьвает, что в случае, когда задано нетривиальное поле симметрий, то теорема 3 и теорема 5 не позволяют найти интеграл, функционально независимьй с интегралом $I(x)$ из следствия 2 . Но несмотря на это, они позволяют находить нетривиальные интегралы и поля симметрии геодезических потоков, когда задан дискретньй траекторный автоморфизм этих потоков. Аналогичные по духу результаты, но другие, получил $\Phi$. А. Магри (см. [4]).

4. Тензорные инварианты на компактных поверхностях постоянной отрицательной кривизны. Пусть $M^{2}$ - компактное ориентированное и замкнутое риманово многообразие без края. Допустим, что оно является пространством постоянной отрицательной кривизны. Тогда согласно следствию 1 на изоэнергетической поверхности $Q^{3}$ все тензорные инварианты типа $(0,1)$ и $(1,0)$ тривиальные, т.е. они имеют соответственно вид const $\Theta^{1}$ и const $D_{1}$.

Рассмотрим теперь случай тензорных инвариантов типа $(0,2),(1,1)$ и $(2,0)$. Нетрудно заметить, что за исключением тензорных инвариантов const $\Theta^{1} \otimes \Theta^{1}$, const $D_{1} \otimes \Theta^{1}$, const $\Theta^{1} \otimes D_{1}$ и const $D_{1} \otimes D_{1}$ нет других, которые бы имели "цельй множитель" $D_{1}$ или $\Theta^{1}$. Допустим, что дан тензорньй инвариант $\omega^{2}$, который порождается формами $T$ и $\Theta^{2}$. Тогда его "коэффициенты” удовлетворяют системе $(* * *)$. Рассмотрим матрицу этой системы

$$
\left[K_{2}\right] \stackrel{\text { def }}{=}\left[\begin{array}{cccc}
0 & -1 & -1 & 0 \\
K & 0 & 0 & -1 \\
K & 0 & 0 & -1 \\
0 & K & K & 0
\end{array}\right] .
$$

Эта матрица имеет собственные значения $\lambda_{1,2}=0, \lambda_{3,4}= \pm \sqrt{-2 K}$. Тогда так как $M$ компактно, в силу ограниченности функций $b_{i j}$ столбец $\left(b_{00}, b_{01}, b_{10}, b_{11}\right)^{T}$ принадлежит $\operatorname{Ker}\left[K_{2}\right]$. Следовательно, существуют гладкие функции $f$ и $g$ такие, что

$$
\omega^{2}=f T \wedge \Theta^{2}+g\left(T \otimes T+K \Theta^{2} \otimes \Theta^{2}\right) .
$$

Так как все формы в этом равенстве инварианты, то $f$ и $g$ являются интегралами; следовательно, они константы.

Случай тензорных инвариантов типа $(1,1)$ и $(2,0)$ сводится к уже рассмотренному "снятием индекса" с помощью формы $\omega_{g}=T \wedge \Theta^{1}$. Тем самым доказано

УТВЕРЖДЕНИЕ 4. Если $M$ - компактное пространство постоянной отрицательной кривизны, то

а) все тензорные инварианты типа $(0,2)$ являются линейной комбиначией с постоянными коэффиииентами форм: $\Theta^{1} \otimes \Theta^{1}, T \wedge \Theta^{2} u\left(T \otimes T+K \Theta^{2} \otimes \Theta^{2}\right)$;

б) все тензорные инварианты типа $(1,1)$, с точностью до изменения порядка сомножителей, являются линейной комбинацией с постоянными коэффициентами операторов: $D_{1} \otimes \Theta^{1}, \mathrm{id},\left(D_{2} \otimes T-K D_{0} \otimes \Theta^{2}\right)$;

в) все тензорные инварианты типа $(2,0)$ являются линейной комбинацией $c$ постоянными коэффициентами тензоров: $D_{1} \otimes D_{1}, D_{0} \wedge D_{2},\left(D_{2} \otimes D_{2}+K D_{0} \otimes D_{0}\right)$. 
5. Тензорные инварианты типа $(0,2)$. Пусть на компактной изоэнергетической поверхности $Q^{3}$ дан тензорньй инвариант геодезического потока типа $(0,2)$. Следует ли отсюда, что этот геодезический поток интегрируем? Конечно, мы заранее должны исключить тривиальные тензорные инварианты $\Theta^{1} \otimes \Theta^{1}$ и $\omega_{g}$, которые всегда имеются. Нами ранее было замечено, что если кривизна $K$ постоянна, то форма $T \otimes T+K \Theta^{2} \otimes \Theta^{2}$ является тензорным инвариантом. Следовательно, если в этом случае $K<0$, то нет не только нетривиальных интегралов, но и ненулевых многозначных интегралов.

ТЕОрема 7. Пусть на компактной изоэнергетической поверхности $Q^{3}$ геодезического потока задан тензорный инвариант $B$ типа $(0,2)$, который равен нулю на конечном числе замкнутых траекторий. Тогда существует двулистное накрытие над дополнением $\kappa$ этим траекториям такое, что поднятый на нем поток имеет ненулевой многозначный интеграл.

ДокАЗАТЕЛЬСтво. Без ограничения общности можно считать, что $B$ порождается формами $T$ и $\Theta^{2}$. В противном случае мы имели бы многозначньй интеграл на $Q^{3}$, так как наличие члена const $\Theta^{1} \otimes \Theta^{1}$ невозможно из-за того, что $B$ в некоторой точке зануляется. Следовательно,

$$
B=b_{00} T \otimes T+b_{01} T \otimes \Theta^{2}+b_{10} \Theta^{2} \otimes T+b_{11} \Theta^{2} \otimes \Theta^{2} .
$$

Это тензорное поле однозначно разбивается в сумму симметрического и кососимметрического, которые, со своей стороны, тоже являются тензорными инвариантами. Следовательно, мы без ограничения общности можем считать, что $B$ - симметрическое тензорное поле. "Поднимая первый индекс с помощью симплектической структуры", по $B$ построим оператор $\widetilde{B}$, которьй снова будет тензорным инвариантом. Имеем

$$
\widetilde{B}=-b_{00} D_{2} \otimes T-b_{01} D_{2} \otimes \Theta^{2}+b_{10} D_{0} \otimes T+b_{11} D_{0} \otimes \Theta^{2} .
$$

Рассмотрим матрищу этого оператора в репере $\left(D_{0}, D_{2}, D_{1}\right)$ :

$$
[\widetilde{B}]=\left[\begin{array}{ccc}
b_{10} & b_{11} & 0 \\
-b_{00} & b_{01} & 0 \\
0 & 0 & 0
\end{array}\right]
$$

Так как в процессе эволюции оператор $\widetilde{B}$ испытывает изоспектральную деформацию, его собственные значения сохраняются. Следовательно, число $b_{00} b_{11}-b_{10} b_{01}$ является интегралом. Если этот интеграл нетривиален, то теорема доказана. Допустим, он равняется константе. Тогда, очевидно, $b_{00} b_{11}-b_{10} b_{01}=0$ тождественно. Рассмотрим ограничение $\widetilde{B}$ на подпространство $\operatorname{Ker} \Theta^{1}$. Обозначим его матрицу снова через [ $\left.\widetilde{B}\right]$,

$$
[\widetilde{B}]=\left[\begin{array}{cc}
b_{10} & b_{11} \\
-b_{00} & -b_{01}
\end{array}\right]
$$

Непосредственно проверяется, что $[\widetilde{B}]^{2}=-\operatorname{det}[\widetilde{B}] I$, где $I-$ единичная матрища. Но так как $\operatorname{det}[\widetilde{B}]=0$, оператор $\widetilde{B}$ нильпотентен. Рассмотрим одномерное инвариантное относительно геодезического потока подпространство $\operatorname{Im} \widetilde{B}=\operatorname{Ker} \widetilde{B}$. Дополнение к окружностям, на которых $\widetilde{B}$ обращается в нуль, обозначим через $Q_{0}^{3}$. На нем $\operatorname{Ker} \widetilde{B}$ задает 
одномерное подрасслоение касательного расслоения $\mathcal{T} Q_{0}^{3}$. Рассмотрим пространство единичных векторов расслоения $\operatorname{Ker} \widetilde{B}$ и обозначим его через $\widetilde{Q}_{0}^{3}$. Конечно, $\widetilde{Q}_{0}^{3}$ двулистно накрывает $Q_{0}^{3}$ (для удобства мы не предполагаем, что накрытие связно). Обозначим естественную проекцию этого накрытия через $\pi: \widetilde{Q}_{0}^{3} \rightarrow Q_{0}^{3}$. Теперь поднимем все эти объекты на $\widetilde{Q}_{0}^{3}$. Для удобства полученные новые геометрические объекты будем обозначать теми же буквами. Тогда расслоение $\operatorname{Ker} \widetilde{B}$ - ориентируемо. Выберем на нем ориентацию и рассмотрим векторное поле единичной длины, которое задает эту ориентацию. Обозначим его через $Z$. В качестве метрики на $Q^{3}$ берем ту метрику, в которой репер $\left(D_{0}, D_{1}, D_{2}\right)$ - ортонормированный. Обозначим ее через $g_{1}$.

Рассмотрим теперь оператор $Z \otimes i_{Z} \omega_{g}$. Так как он, очевидно, нильпотентен и имеет такое же ядро, как и $\widetilde{B}$, то эти операторы пропорциональны на $\widetilde{Q}_{0}^{3}$, т.е. существует нигде не зануляющаяся гладкая функция $\psi$ такая, что $\widetilde{B}=\psi Z \otimes i_{Z} \omega_{g}$. Теперь докажем, что векторное поле $X \stackrel{\text { def }}{=} \sqrt{|\psi|} Z$ является полем симметрии поднятого на $\widetilde{Q}_{0}^{3}$ геодезического потока.

Действительно, пусть $\left\{g^{t}(x)\right\}$ - геодезический поток на $\widetilde{Q}_{0}^{3}$. Тогда в силу инвариантности $\widetilde{B}$ и $\omega_{g}$ имеем

$$
\begin{aligned}
\widetilde{B}(x)(\cdot) & =\left[d_{x} g^{t}\right]^{-1} B\left(g^{t}(x)\right)\left(d_{x} g^{t}(\cdot)\right) \\
& =\varepsilon\left[d_{x} g^{t}\right]^{-1}(X) \otimes \omega_{g}\left(g^{t}(x)\right)\left(X, d_{x} g^{t}(\cdot)\right) \\
& =\varepsilon\left[d_{x} g^{t}\right]^{-1}(X) \otimes \omega_{g}(x)\left(\left[d_{x} g^{t}\right]^{-1}(X), \cdot\right),
\end{aligned}
$$

где $\varepsilon$ обозначает знак функции $g$. С другой стороны, очевидно $\widetilde{B}(x)=\varepsilon X(x) \otimes$ $\omega_{g}(x)(X(x), \cdot)$. Следовательно, $\left[d_{x} g^{t}\right]^{-1}\left(X\left(g^{t}(x)\right)\right)= \pm X(x)$. В силу непрерывности получаем, что $X$ - поле симметрии.

Теперь докажем, что на $\widetilde{Q}_{0}^{3}$ имеется многозначный интеграл.

Пусть $X=x D_{2}+y D_{0}$, где $x$ и $y$ гладкие функции на $\widetilde{Q}_{0}^{3}$. $\underset{\widetilde{Q}}{\mathrm{C}}$ другой стороны, нетрудно заметить, что определенная ранее гладкая функция $\psi$ на $\widetilde{Q}_{0}^{3}$ на самом деле является гладкой функцией на $Q_{0}^{3}$. Это следует из того, что уравнение $\widetilde{B}=\psi Z \otimes i_{Z} \omega_{g}$ инвариантно при замене $Z \mapsto-Z$. Из этого уравнения также видно, что функцию $\psi$ можно доопределить до непрерьвной на всем $Q^{3}$, полагая $\psi=0$ там, где $\widetilde{B}=0$. Следовательно, $\psi$ - ограниченная функция на $Q^{3}$, а следовательно, и на $\widetilde{Q}_{0}^{3}$. Таким образом, получаем, что $|X|_{1}=|\sqrt{|\psi|} Z|_{1}=|\psi|<M_{0}$. В частности, $|x|<M_{0}$ и $|y|<M_{0}$ на $\widetilde{Q}_{0}^{3}$. Как и ранее, рассмотрим форму $\alpha_{X} \stackrel{\text { def }}{=} i_{X} \omega_{g}=-x T+y \Theta^{2}$. Тогда $d \alpha_{X}=\left[y_{0}+x_{2}\right] T \wedge \Theta^{2}$. Следовательно, $d\left[\Theta^{1} \wedge \alpha_{X}\right]=\left[y_{0}+x_{2}\right] T \wedge \Theta^{1} \wedge \Theta^{2}$. Допустим, что интеграл $I \stackrel{\text { def }}{=} y_{0}+x_{2}$ тривиален, т.е. $I=c=$ const. Имеем равенство

$$
d\left[x T \wedge \Theta^{1}+y \Theta^{1} \wedge \Theta^{2}\right]=c T \wedge \Theta^{1} \wedge \Theta^{2} .
$$

Ориентацию на $\widetilde{Q}_{0}^{3}$ зададим формой $\sigma_{g} \stackrel{\text { def }}{=} T \wedge \Theta^{1} \wedge \Theta^{2}$. Обозначим теперь те траектории, на которых $\widetilde{B}=0$, через $\left\{S_{i}^{1}\right\}_{i=1}^{N}$. Рассмотрим их трубчатые окрестности в $Q^{3}$.

ЛЕмма 1. В окрестности любой траектории $S_{i}^{1}$ существует последовательность трубчатых окрестностей (полноторий) $\left\{T_{i}(n)\right\}_{n=1}^{\infty}$, которые удовлетворяют условиям:

$$
T_{i}(n) \supseteq T_{i}(n+1),
$$

2) $\operatorname{Vol}\left(T_{i}(n)\right) \rightarrow 0 \quad(n \rightarrow 0)$,

3) $\operatorname{Vol}\left(\partial T_{i}(n)\right) \rightarrow 0 \quad(n \rightarrow 0)$. 
Здесь в качестве оббема берем римановый оббем, индучированный метрикой $g_{1}$.

ДоКАЗАТЕЛЬСТво не составляет труда.

Рассмотрим последовательность многообразий $Q^{3}(n) \stackrel{\text { def }}{=} Q^{3} \backslash \bigsqcup_{i} T_{i}(n)$ и их двулистные накрытия $\widetilde{Q}^{3}(n) \stackrel{\text { def }}{=} \pi^{*} Q^{3}(n) \supseteq \widetilde{Q}_{0}^{3}$. Проинтегрируем теперь равенство (3) по $\widetilde{Q}^{3}(n)$. Получаем

$$
c \operatorname{Vol} \widetilde{Q}^{3}(n)=\sum_{i=1}^{n} \int_{\partial T_{i}(n)}\left[x T \wedge \Theta^{2}+y \Theta^{1} \wedge \Theta^{2}\right],
$$

где $\widetilde{\partial T_{i}(n)} \stackrel{\text { def }}{=} \pi^{*}\left(\partial T_{i}(n)\right)$ ориентированные "внешней" нормалью. Следовательно,

$$
c 2 \operatorname{Vol} Q^{3}=2 c \sum_{i=1}^{N} \operatorname{Vol} T_{i}(n)+\sum_{i=1}^{N} \int_{\overparen{\partial T_{i}(n)}}\left[x T \wedge \Theta^{2}+y \Theta^{1} \wedge \Theta^{2}\right] .
$$

Оценим вторую компоненту суммы в правой части этого равенства:

$$
\begin{aligned}
& \left|\sum_{i=1}^{N} \int_{\widetilde{\partial T_{i}(n)}}\left[x T \wedge \Theta^{2}+y \Theta^{1} \wedge \Theta^{2}\right]\right| \\
& \quad \leqslant \sum_{i=1}^{N}\left\{\int_{\widetilde{\partial T_{i}(n)}}|x|\left|T \wedge \Theta^{2}\right|+\int_{\widetilde{\partial T_{i}(n)}}|y|\left|\Theta^{1} \wedge \Theta^{2}\right|\right\} \\
& \quad \leqslant M_{0} \sum_{i=1}^{N}\left\{\int_{\widetilde{\partial T_{i}(n)}}\left|T \wedge \Theta^{2}\right|+\int_{\widetilde{\partial T_{i}(n)}}\left|\Theta^{1} \wedge \Theta^{2}\right|\right\} \\
& \quad=2 M_{0} \sum_{i=1}^{N}\left\{\int_{\partial T_{i}(n)}\left|T \wedge \Theta^{2}\right|+\int_{\partial T_{i}(n)}\left|\Theta^{1} \wedge \Theta^{2}\right|\right\} .
\end{aligned}
$$

Рассмотрим проективизацию касательного расслоения $P \stackrel{\text { def }}{=} P\left(\mathcal{T} Q^{3}\right) \cong Q^{3} \times R P^{2}$. Это, конечно, компактное многообразие. Тогда любая из форм $\left|T \wedge \Theta^{2}\right|$ и $\left|\Theta^{1} \wedge \Theta^{2}\right|$ задает гладкую функцию на $P$ формулой,

$$
\left\{l-\text { прямая в } \mathcal{T}_{x} Q^{3}\right\} \mapsto\left|T \wedge \Theta^{2}(\xi, \eta)\right|,
$$

где $\xi, \eta \in \mathcal{T}_{x} Q^{3}$ ортонормированные в плоскости $L$, ортогональной к $l$. Рассмотрим константу

$$
M_{1} \stackrel{\text { def }}{=} \max \left\{\max _{p}\left|T \wedge \Theta^{2}\right|, \max _{p}\left|\Theta^{1} \wedge \Theta^{2}\right|\right\} .
$$

Тогда, очевидно, имеем оценки

Следовательно,

$$
\int_{\partial T_{i}(n)}\left|T \wedge \Theta^{2}\right| \leqslant M_{1} \operatorname{Vol}\left(\partial T_{i}(n)\right)
$$

и

$$
\int_{\partial T_{i}(n)}\left|\Theta^{1} \wedge \Theta^{2}\right| \leqslant M_{1} \operatorname{Vol}\left(\partial T_{i}(n)\right) .
$$

$$
\left|\sum_{i=1}^{N} \int_{\partial T_{i}(n)}\left[x T \wedge \Theta^{2}+y \Theta^{1} \wedge \Theta^{2}\right]\right| \leqslant 4 M_{0} M_{1} \sum_{i=1}^{N} \operatorname{Vol}\left(\partial T_{i}(n)\right) .
$$

Теперь, устремляя $n \rightarrow \infty$ в равенстве (4), получаем, что $c=0$. Следовательно, $\alpha_{X}-$ многозначньй интеграл на $\widetilde{Q}_{0}^{3}$. Тем самым, теорема доказана. 
6. Тензорные инварианты типа $(m, n)$. Допустим, что геодезический поток на ориентируемой двумерной поверхности допускает тензорный инвариант $B$ типа $(m, n)$ на изоэнергетической поверхности $Q^{3}$. Допустим кроме этого, что в некоторой точке поле $B$ обращается в нуль. Докажем, что в этом случае геодезический поток допускает в некотором естественном смысле “двузначное” нетривиальное поле симметрий. Точный смысл этого понятия будет точно сформулирован в дальнейшем.

Мы без ограничения общности можем считать, что тензорное поле $B$ порождается полями $D_{0}, D_{2}, T$ и $\Theta^{2}$ (т.е. $B$ является конечной суммой мономов, которые являются тензорными произведениями таких полей). В противном случае, свертывая $D_{1}$ с $\Theta^{1}$, мы получим тензорный инвариант, который в некоторой точке равен нулю и который порождается требуемыми полями. С другой стороны, пользуясь формой $\omega_{g}=T \wedge \Theta^{2}$, мы можем без ограничения общности считать, что $B$ является полилинейной формой. Рассмотрим теперь инвариантное относительно геодезического потока тензорное поле $P \stackrel{\text { def }}{=} D_{0} \wedge D_{2}$. Если допустим, что тензор $B$ несимметричен, то, свертьвая его с $P$ по соответствуюшим аргументам, получим тензорный инвариант на два порядка ниже, который в некоторой точке на $Q^{3}$ равен нулю. Допуская, что он тоже несимметричен, и применяя эту процедуру еще несколько раз, мы в конечном итоге получим тензорный инвариант, который является симметрическим тензором. Далее мы будем считать, что $B$ является симметрическим тензорным инвариантом типа $(0, d)$, т.е.

$$
B=b_{i_{1} \ldots i_{d}} T^{i_{1}} \otimes \cdots \otimes T^{i_{d}} \quad\left(i_{k}=0,1\right)
$$

где $T^{0} \stackrel{\text { def }}{=} T$ и $T^{1} \stackrel{\text { def }}{=} \Theta^{2}$, a $\left(b_{i_{1} \ldots i_{d}}\right)$ - гладкие функции на $Q^{3}$ и $b_{i_{1} \ldots i_{d}}=b_{i_{\sigma}(1) \ldots i_{\sigma}(d)}$ $\left(\sigma\right.$ пробегает группу перестановок $S_{d}$ ).

Рассмотрим отображение $\mathcal{B}: Q^{3} \rightarrow R^{d+1}$, которое зададим формулой

$$
\mathcal{B}(x) \stackrel{\text { def }}{=}\left(b_{i_{1} \ldots i_{d}}(x)\right)
$$

Пусть $x_{0} \in Q^{3}$. Рассмотрим геодезическую траекторию $\phi^{t} x_{0}$ и некоторую точку $y_{0}$, лежащую на ней, т.е. $y_{0}=\phi^{t_{0}} x_{0}$.

Рассмотрим отображение “тривиализации"

$$
\tau: \mathcal{T} Q^{3} \rightarrow R^{3}
$$

которое зададим формулой

$$
\xi_{x_{0}} \mapsto\left(T\left(\xi_{x_{0}}\right), \Theta\left(\xi_{x_{0}}\right), \Theta^{1}\left(\xi_{x_{0}}\right)\right)
$$

Рассмотрим коммутативную диаграмму

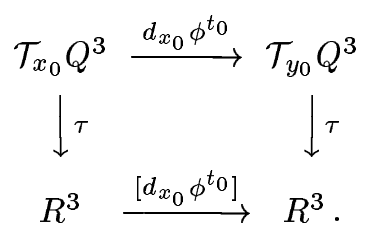


Тогда матрица $\left[d_{x_{0}} \phi^{t_{0}}\right]$ имеет вид

$$
\left[d_{x_{0}} \phi^{t_{0}}\right] \stackrel{\text { def }}{=}\left[\begin{array}{ccc}
a_{1}^{1} & a_{2}^{1} & 0 \\
a_{1}^{2} & a_{2}^{2} & 0 \\
0 & 0 & 1
\end{array}\right]
$$

где $a_{1}^{1} a_{2}^{2}-a_{2}^{1} a_{1}^{2}=1$ (так как $\left\{\phi^{t} x\right\}_{t}$ сохраняет форму $\left.\omega_{g} \stackrel{\text { def }}{=} T \wedge \Theta^{2}\right)$. Следовательно, формы $\mathcal{B}\left(x_{0}\right)$ и $\mathcal{B}\left(y_{0}\right)$ связаны между собой соотношением

$$
\mathcal{B}\left(y_{0}\right)=\rho(A)\left(\mathcal{B}\left(x_{0}\right)\right)
$$

где $A \stackrel{\text { def }}{=}\left(a_{j}^{i}\right) \in S L_{2}(R)$, а $\rho$ есть линейное представление

$$
\rho: S L_{2}(R) \rightarrow G L\left(R^{d+1}\right), R^{d+1} \cong S^{d}\left(R^{2^{*}}\right), R^{2^{*}} \stackrel{\text { def }}{=}\left\langle d x^{1}, d x^{2}\right\rangle_{R}
$$

которое задается формулой

$$
[\rho(A)(B)]_{j_{1} \ldots j_{d}} \stackrel{\text { def }}{=} \tilde{a}_{j_{1}}^{i_{1}} \ldots \tilde{a}_{j_{d}}^{i_{d}} b_{i_{1} \ldots i_{d}}, \quad\left(\tilde{a}_{j}^{i}\right) \stackrel{\text { def }}{=} A^{-1} .
$$

Следовательно, кривая

$$
t \mapsto \mathcal{B}\left(\phi^{t}\left(x_{0}\right)\right) \in R^{d+1}
$$

лежит на одной орбите действия $\rho$.

Допустим теперь, что $I_{\alpha}$ - некоторьй инвариант действия $\rho$ (т.е. $I_{\alpha}$ - гладкая функция от координат $\left(b_{i_{1}} \ldots i_{d}\right)$, которая постоянна на орбитах действия $\left.\rho\right)$. Тогда, очевидно, сквозное отображение $\mathcal{I}_{\alpha} \stackrel{\text { def }}{=} I_{\alpha} \circ \mathcal{B}$

$$
\begin{array}{crr}
R^{d+1} & & \\
\uparrow_{\mathcal{B}} & \searrow \\
Q^{3} & \stackrel{\mathcal{I}_{\alpha}}{I_{\alpha}} & R^{1}
\end{array}
$$

будет интегралом геодезического потока. Предположим, что инвариант $I_{\alpha}$ является однородным многочленом от координат $\left(b_{i_{1}} \ldots i_{d}\right)$. Тогда, если интеграл $\mathcal{I}_{\alpha}=$ const, то $\mathcal{I}_{\alpha} \equiv 0$ (так как в некоторой точке $z_{0} \in Q^{3}$ по условию, имеем $\left.\mathcal{B}\left(z_{0}\right)=0\right)$. Следовательно, без ограничения общности можно считать, что $\mathcal{I}_{\alpha} \equiv 0$ на $Q^{3}$, когда $I_{\alpha}$ пробегает множество всех однородных инвариантов положительной степени. В противном случае мы нашли бы нетривиальньй интеграл.

Имеет место следующая лемма, которая является простым следствием из критерия Гильберта (см. $[5, \S 5])$. 
Лемма 2. Пусть форма $B \in S^{d}\left(R^{2^{*}}\right)$ является нильпотентным әлементом действия $\rho$. Рассмотрим последовательность полилинейных форм

$$
\begin{aligned}
& P_{0} \stackrel{\text { def }}{=} B \otimes B, \\
& P_{k} \stackrel{\text { def }}{=} C\left(\widetilde{\omega} \otimes P_{k-1}\right) \quad(k=1,2, \ldots),
\end{aligned}
$$

где С обозначает свертку $\widetilde{\omega}$ по первому и последнему аргументу формы $P_{k-1}$. Очевидно, существует иелое число $l$ такое, что $P_{l} \neq 0$ u $P_{l+1}=0$. Тогда существует форма $f$ такая, что

$$
P_{l}=f^{\otimes 2 p}, \quad p \geqslant 1 \text {. }
$$

Пусть теперь $B$ - симметрический тензорньй инвариант типа $(0, d)$. Как было замечено ранее, без ограничения общности можно считать, что все интегралы $\mathcal{I}_{\alpha}(x) \stackrel{\text { def }}{=} I_{\alpha}$ 。 $\mathcal{B}(x)$, когда $I_{\alpha}$ пробегает множество всех однородных инвариантов положительной степени, тождественно равны нулю. Следовательно, формы $B(x)$ во всех точках $x \in Q^{3}$ являются нульформами. Обозначим через $m$ максимальную степень вырождения форм $B(x)$, когда $x$ пробегает все $Q^{3}$. Рассмотрим теперь тензорньй инвариант $P_{2(d-m)}(x)$. Согласно доказанной лемме это симметрическое тензорное поле типа $(0,2(2 m-d))$ такое, что

$$
P_{2(d-m)}(x)=f(x)^{\otimes 2(2 m-d)},
$$

где $f(x)$ "натянуто" на $T_{x}$ и $\Theta_{x}^{2}$ (мы пока никакой гладкости сечения $f(x)$ по $x$ не предполагаем).

Обозначим через $N$ множество точек из $Q^{3}$, в которых $P_{2(d-m)}=0$. Очевидно, что $N \neq \varnothing$. Так же, как и в доказательстве теоремы 7 , доказывается, что:

а) если $x_{0} \in Q^{3} \backslash N$, то существует окрестность $U\left(x_{0}\right)$ точки $x_{0}$ и гладкая 1-форма $f$ на ней, для которой выполнено равенство (6); очевидно, 1-форма $(-f)$ тоже удовлетворяет (6);

б) множество решений $f$ уравнения (6) инвариантно относительно геодезического потока;

в) пусть $g_{1}$ - определенная в п. 5 риманова метрика на $Q^{3}$. Распространим ее на тензорные поля. Тогда из равенства (6) получаем, что

$$
|f|_{1}=\left(\left|P_{2(d-m)}\right| 1\right)^{1 /(2 m-d)},
$$

и, следовательно, норма $f$ является гладкой функцией на $Q^{3}$.

ОПРЕДЕЛЕНИЕ 3. Пусть $\mathcal{F}$ - подпространство $\mathcal{T}^{*} Q^{3}$, а $\pi: \mathcal{F} \rightarrow Q^{3}$ - естественная проекция. Обозначим через $N_{\mathcal{F}}$ множество тех точек $x \in Q^{3}$, для которых $\pi^{-1}(x)=$ $\{0\}$. Предположим, что

i) ограничение отображения $\pi$ на множество $\pi^{-1}\left(Q^{3} \backslash N_{\mathcal{F}}\right)$ является $k$-листным гладким накрытием (гладкость понимается в том смысле, что локально любой лист является гладким сечением в $\left.\mathcal{T}^{*} Q^{3}\right)$;

ii) множество $\mathcal{F}$ инвариантно относительно геодезического потока, т.е. если $f \in \mathcal{F}$, то $\phi_{t}^{*} f \in \mathcal{F}$; 
iii) если $g_{1}-$ произвольная гладкая метрика на $Q^{3}$, то функция

$$
|\mathcal{F}(x)|_{1} \stackrel{\text { def }}{=} \max \left\{\left|f_{x}\right|_{1}: f_{x} \in \pi^{-1}(x)\right\}
$$

должна быть гладкой.

Если выполнено i)-iii), то $\mathcal{F}$ будем называть $k$-значным полем симметрий геодезического потока.

\section{Тем самым доказана}

ТЕОРема 9. Пусть геодезический поток двумерной поверхности допускает на изоэнергетической поверхности $Q^{3}$ тензорный инвариант типа $(m, n)$, который в некоторой точке равен нулю. Тогда геодезический поток допускает нетривиальное двузначное поле симметрий.

Автор пользуется случаем выразить свою признательность А. Т. Фоменко, В. В. Козлову, Э.Б. Винбергу и А. В. Болсинову за полезные обсуждения.

\section{СПИСОК ЦИТИРОВАННОЙ ЛИТЕРАТУРЫ}

[1] Козлов В. В. Симметрии, топология и резонансы в гамильтоновой механике. Ижевск: УдГУ, 1995.

[2] Bolotin S. V., Kozlov V. V. Symmetry fields of geodesic flows // Russian J. of Math. Phil.. (to appear).

[3] Арнольд В. И. Математические методы классической механики. М.: Наука, 1974.

[4] Fomenko A. T. Integrability and Nonintegrability in Geometry and Mechanics: Kluwer Acad. Publ., 1988.

[5] Винберг Э. Б., Попов В.Л. Теория инвариантов // Итоги науки и техники. Современные проблемы математики. Фундаментальные направления. Т. 55. М.: Наука, 1989. C. $137-309$. 